\title{
SEARCH FOR A CORRELATION BETWEEN ANTARES NEUTRINOS AND PIERRE AUGER OBSERVATORY UHECRS ARRIVAL DIRECTIONS
}

\begin{abstract}
S. Adrián-Martínez ${ }^{1}$, I. Al Samarai ${ }^{2}$, A. Albert ${ }^{3}$, M. André $^{4}$, M. Anghinolfit ${ }^{5}$, G. Anton ${ }^{6}$, S. Anvar ${ }^{7}$, M. Ardid $^{1}$, T. Astraatmadja ${ }^{8.38}$, J-J. Aubert ${ }^{2}$, B. Baret ${ }^{9}$, S. Basa ${ }^{111}$, L. J. BeEmSTer ${ }^{8}$, V. Bertin ${ }^{2}$, S. Biagi ${ }^{11,12}$, C. Bigongiari ${ }^{13}$, C. Bogazzi ${ }^{8}$, M. Bou-Cabo ${ }^{1}$, B. Bouhou ${ }^{9}$, M. C. Bouwhuis ${ }^{8}$, J. Brunner ${ }^{2,39}$, J. Busto ${ }^{2}$, F. Camarena ${ }^{1}$, A. Capone ${ }^{14,15}$, C. Cârlogand ${ }^{16}$, G. Carminati ${ }^{11,12,41}$, J. Carr ${ }^{2}, \mathrm{~S}$. CeCChini ${ }^{11}$, Z. Charif $^{2}$, Ph. Charvis $^{17}$, T. Chiarusi $^{11}$, M. CirCella $^{18}$,

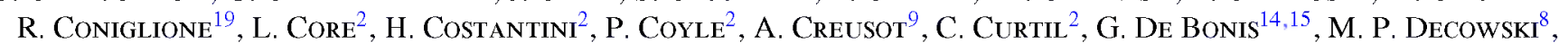
I. DeKeyser ${ }^{21}$, A. Deschamps ${ }^{17}$, C. Distefano ${ }^{19}$, C. DonZaud ${ }^{9.21}$, D. Dornic ${ }^{2}$, Q. Dorosti ${ }^{22}$, D. Drouhin ${ }^{3}$, T. EberL ${ }^{6}$,

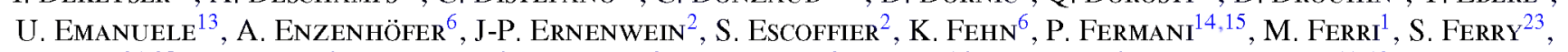
V. Flaminio ${ }^{24,25}$, F. Folger ${ }^{6}$, U. Fritsch ${ }^{6}$, J-L. Fuda ${ }^{21}$, S. Galatà ${ }^{2}$, P. Gay $^{16}$, K. Geyer ${ }^{6}$, G. Giacomellit ${ }^{11,12}$, V. Giordano ${ }^{19}$, J. P. GómeZ-González ${ }^{13}$, K. GraF ${ }^{6}$, G. Guillard ${ }^{16}$, G. Halladjian $^{2}$, G. Hallewell ${ }^{2}$, H. van Haren ${ }^{26}$, J. HarTman ${ }^{8}$, A. J. HeiJBOeR ${ }^{8}$, Y. Hello ${ }^{17}$, J. J. HernÁNDEZ-ReY ${ }^{13}$, B. Herold ${ }^{6}$, J. HÖßL ${ }^{6}$, C. C. HSU ${ }^{8}$, M. DE JONG ${ }^{8,38}$, M. KAdLER ${ }^{27}$,

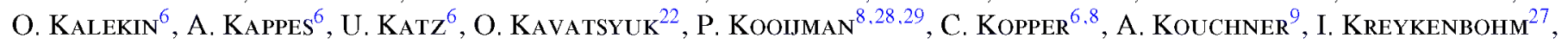

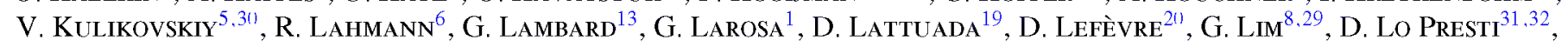

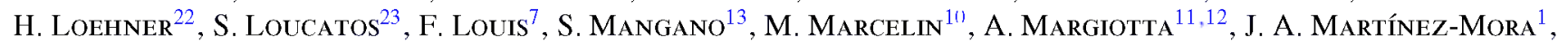

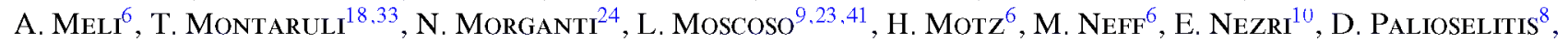
G. E. Păvălaş ${ }^{34}$, K. Payet ${ }^{23}$, P. Payre ${ }^{2,41}$, J. Petrovic ${ }^{8,42}$, N. Picot-Clemente ${ }^{2}$, V. Popa ${ }^{34}$, T. Pradier ${ }^{35}$, E. Presani ${ }^{8}$,



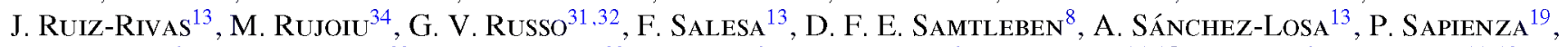

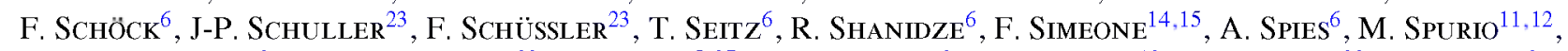

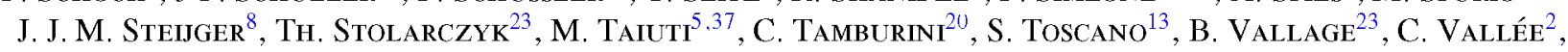

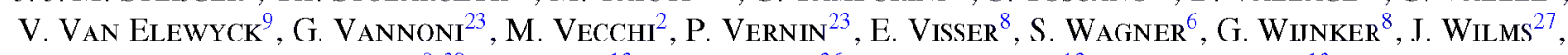
E. DE WOLF ${ }^{8,29}$, H. YePES $^{13}$, D. ZABOROV ${ }^{36}$, J. D. ZORNOZA ${ }^{13}$, AND J. ZÚÑIGA ${ }^{13}$

${ }^{1}$ Institut d'Investigació per a la Gestió Integrada de les Zones Costaneres (IGIC), Universitat Politècnica de València, C/ Paranimf 1 , E-46730 Gandia, Spain ${ }^{2}$ CPPM, Aix-Marseille Université, CNRS/IN2P3, F-13288 Marseille, France

${ }^{3}$ GRPHE-Institut universitaire de technologie de Colmar, 34 rue du Grillenbreit BP 50568-68008 Colmar, France

${ }^{4}$ Laboratory of Applied Bioacoustics, Technical University of Catalonia, Rambla Exposició, E-08800 Vilanova i la Geltrú, Barcelona, Spain 5 INFN-Sezione di Genova, Via Dodecaneso 33, I-16146 Genova, Italy

${ }^{6}$ Friedrich-Alexander-Universität Erlangen-Nürnberg, Erlangen Centre for Astroparticle Physics, Erwin-Rommel-Str. 1, D-91058 Erlangen, Germany

${ }^{7}$ Direction des Sciences de la Matière, Institut de recherche sur les lois fondamentales de 1'Univers, Service d'Electronique des Détecteurs et d'Informatique, CEA Saclay, F-91191 Gif-sur-Yvette Cedex, France

8 Nikhef, Science Park, Amsterdam, The Netherlands

${ }^{9}$ APC-Laboratoire AstroParticule et Cosmologie, UMR 7164 (CNRS, Université Paris 7 Diderot, CEA, Observatoire de Paris) 10, rue Alice Domon et Léonie Duquet, F-75205 Paris Cedex 13, France

${ }^{10}$ LAM-Laboratoire d'Astrophysique de Marseille, Pôle de l'Étoile Site de Château-Gombert, rue Frédéric Joliot-Curie 38, F-13388 Marseille Cedex 13, France ${ }^{11}$ INFN-Sezione di Bologna, Viale Berti-Pichat 6/2, I-40127 Bologna, Italy

12 Dipartimento di Fisica dell'Università, Viale Berti Pichat 6/2, I-40127 Bologna, Italy

${ }^{13}$ IFIC-Instituto de Física Corpuscular, Edificios Investigación de Paterna CSIC, Universitat de València, Apdo. de Correos 22085 , E-46071 Valencia, Spain ${ }^{14}$ INFN-Sezione di Roma, P.le Aldo Moro 2, I-00185 Roma, Italy

15 Dipartimento di Fisica dell'Università La Sapienza, P.le Aldo Moro 2, I-00185 Roma, Italy

${ }^{16}$ Clermont Université, Université Blaise Pascal, CNRS/IN2P3, Laboratoire de Physique Corpusculaire, BP 10448 , F-63000 Clermont-Ferrand, France

${ }^{17}$ Géoazur-Université de Nice Sophia-Antipolis, CNRS/INSU, IRD, Observatoire de la Côte d'Azur and Université Pierre et Marie Curie, BP 48, F-06235 Villefranche-sur-mer, France

${ }^{18}$ INFN-Sezione di Bari, Via E. Orabona 4, I-70126 Bari, Italy

${ }^{19}$ INFN-Laboratori Nazionali del Sud (LNS), Via S. Sofia 62, I-95123 Catania, Italy

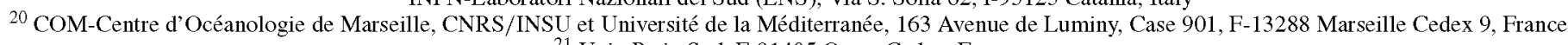
${ }^{21}$ Univ Paris-Sud, F-91405 Orsay Cedex, France

${ }^{22}$ Kernfysisch Versneller Instituut (KVI), University of Groningen, Zernikelaan 25, 9747 AA Groningen, The Netherlands

${ }^{23}$ Direction des Sciences de la Matière-Institut de recherche sur les lois fondamentales de l'Univers, Service de Physique des Particules, CEA Saclay, F-91191 Gif-sur-Yvette Cedex, France

${ }^{24}$ INFN-Sezione di Pisa, Largo B. Pontecorvo 3, I-56127 Pisa, Italy

25 Dipartimento di Fisica dell'Università, Largo B. Pontecorvo 3, I-56127 Pisa, Italy

${ }^{26}$ Royal Netherlands Institute for Sea Research (NIOZ), Landsdiep 4, 1797 SZ 't Horntje (Texel), The Netherlands

${ }^{27}$ Dr. Remeis-Sternwarte and ECAP, Universität Erlangen-Nürnberg, Sternwartstr. 7, D-96049 Bamberg, Germany

${ }^{28}$ Universiteit Utrecht, Faculteit Betawetenschappen, Princetonplein 5, 3584 CC Utrecht, The Netherlands

${ }^{29}$ Universiteit van Amsterdam, Instituut voor Hoge-Energie Fysika, Science Park 105, 1098 XG Amsterdam, The Netherlands

${ }^{30}$ Skobeltsyn Institute of Nuclear Physics, Moscow State University, Leninskie gory, 119991 Moscow, Russia

31 INFN-Sezione di Catania, Viale Andrea Doria 6, I-95125 Catania, Italy

32 Dipartimento di Fisica ed Astronomia dell'Università, Viale Andrea Doria 6, I-95125 Catania, Italy

${ }^{33}$ DPNC, Université de Genève, 1211 Geneva, Switzerland

34 Institute for Space Sciences, R-77125 Bucharest, Măgurele, Romania

${ }^{35}$ IPHC-Institut Pluridisciplinaire Hubert Curien, Université de Strasbourg et CNRS/IN2P3 23 rue du Loess, BP 28 , F-67037 Strasbourg Cedex 2, France

${ }^{36}$ ITEP-Institute for Theoretical and Experimental Physics, B. Cheremushkinskaya 25, 117218 Moscow, Russia

${ }^{37}$ Dipartimento di Fisica dell'Università, Via Dodecaneso 33, I-16146 Genova, Italy

Received 2012 May 23; accepted 2013 June 20; published 2013 August 12 


\begin{abstract}
A multimessenger analysis optimized for a correlation of arrival directions of ultra-high energy cosmic rays (UHECRs) and neutrinos is presented and applied to 2190 neutrino candidate events detected in 2007-2008 by the ANTARES telescope and 69 UHECRs observed by the Pierre Auger Observatory between 2004 January 1 and 2009 December 31. No significant correlation is observed. Assuming an equal neutrino flux ( $E^{-2}$ energy spectrum) from all UHECR directions, a $90 \%$ CL upper limit on the neutrino flux of $5.0 \times 10^{-8} \mathrm{GeV} \mathrm{cm}^{-2} \mathrm{~s}^{-1}$ per source is derived.
\end{abstract}

Key words: astroparticle physics - cosmic rays - neutrinos

Online-only material: color figures

\section{INTRODUCTION}

The astrophysical sources of ultra-high energy cosmic rays (UHECRs) and neutrinos remain unknown. Astrophysical sources expected to produce comparable fluxes of cosmic rays and neutrinos are, for example, the jets of gamma-ray bursts (Waxman 1995; Vietri 1995; Waxman \& Bahcall 1997; Murase et al. 2006) or active galactic nuclei (AGNs; Biermann \& Strittmatter 1987; Rachen \& Biermann 1993; Nellen et al. 1993; Mannheim 1995; Rachen 2008).

The search for UHECR sources is complicated by their deflection in magnetic fields inside and outside of our Galaxy. While the existence of a cut-off in the energy spectrum of UHECRs, first observed by the High Resolution Fly's Eye cosmic-ray detector (HiRes) experiment (Abbasi et al. 2008a, $2009 \mathrm{~b}$ ), has now been confirmed by the data of the Pierre Auger Observatory (Abraham et al. 2008a, 2010), the composition of cosmic rays above a few $10^{18} \mathrm{eV}$, crucial for the estimation of expected magnetic deflection magnitudes, remains uncertain. Although data from the Pierre Auger Observatory seem to indicate a transition from a light to a heavy composition above $40 \mathrm{EeV}$, this trend is still subject to large uncertainties, in particular related to the lack of accurate modeling of hadronic interactions in the relevant energy domain.

Due to their interactions with photons of the cosmic microwave background via the GZK mechanism (Greisen 1966; Zatsepin \& Kuzmin 1966), UHECR propagation distances are limited, for example, the proton energy loss distance above $10^{20} \mathrm{eV}$ is about $100 \mathrm{Mpc}$. This means that only nearby astrophysical sources, lying within the GZK sphere could possibly be identified as UHECR acceleration sites. This has been extensively discussed in the context of model-dependent theoretical upper bounds on neutrino fluxes by Waxman \& Bahcall (1999), Rachen et al. (2000), Mannheim et al. (2001), and Bahcall \& Waxman (2001).

A multimessenger approach to the problem of the identification of UHECR sources is based on the detection of secondary fluxes of gamma-rays and neutrinos associated with the decay of pions resulting from the interaction of UHECRs with matter or photon fields in the vicinity of the cosmic accelerators (Waxman \& Bahcall 1999; Rachen et al. 2000; Mannheim et al. 2001; Bahcall \& Waxman 2001; Becker 2008; Becker \& Biermann 2009). Although gamma-rays have been linked to astrophysical sources by recent observations (HESS, MAGIC, VERITAS, Fermi), an unambiguous identification of these

\footnotetext{
38 Also at University of Leiden. The Netherlands.

39 On leave at DESY, Platanenallee 6, D-15738 Zeuthen, Germany.

${ }^{40}$ Now at University of California. Irvine. CA 92697. USA.

${ }^{41}$ Deceased.

${ }^{42}$ Corresponding author.
}

sources as sites of hadronic acceleration requires the detection of the associated neutrino flux. Neutrinos, being neutral and weakly interacting particles, are neither deflected nor attenuated during their propagation from their sources to the Earth. Their small cross-section for interaction with matter, however, makes their detection challenging and requires the construction of very large telescopes. Indeed, the currently operating neutrino telescopes, ANTARES, IceCube and BAIKAL, have not yet observed any statistically significant cosmic neutrino source (Adrián-Martínez et al. 2011, 2012; Abbasi et al. 2011; Avrorin et al. 2009).

In this paper, the first UHECR-neutrino multimessenger study is presented. A source stacking method optimized for a correlation of arrival directions of UHECRs and neutrinos has been developed and applied on the neutrino candidate events detected by the ANTARES telescope and the UHECR events observed by the Pierre Auger Observatory. If such a correlation were observed, it would indicate regions of the sky where the sources of UHECR and neutrinos could plausibly lie, as well as shed light both on the UHECR composition and on the intensity of magnetic fields in and outside of our Galaxy. An observed correlation would also exclude the possibility that the dominant sources of UHECRs are single-shot transient sources, since the time delay between neutrinos and protons coming from such a source is expected to be orders of magnitude larger than the observation times of the ANTARES telescope and the Pierre Auger Observatory.

It should be noted that not all astrophysical sources where protons are accelerated are necessarily sources of neutrinos. Astrophysical sources already mentioned, which are expected to produce comparable fluxes of neutrinos and cosmic rays, have proton interaction opacity $\tau=D_{\mathrm{s}} \sigma \rho \gtrsim 1$ ( $\sigma$ is the crosssection for proton-photon and proton-proton interactions, $\rho$ is the number density of photons and $D_{\mathrm{s}}$ is the scale dimension of the source).

Astrophysical sources with large proton interaction mean free path (corresponding to $\tau \ll 1$ ) may just accelerate and eject UHECR without producing significant neutrino fluxes. Examples for this class could be large-scale cosmic structure shocks (Kang \& Jones 2005; Ma et al. 2011), accretion shocks around clusters of galaxies (Kang et al. 1996, 1997), or radio galaxy lobes and hot spots (Rachen \& Biermann 1993). In this case, some of the arrival directions of the observed UHECRs would be correlated with the arrival directions of neutrinos, and some would not.

Sources with small proton interaction mean free path $(\tau \gg 1)$ would absorb all accelerated cosmic rays in interactions and only neutrinos would be emitted. A model presented by Stecker et al. (1991) falls into this class. In this case a cosmic neutrino flux (likely isotropic) would be expected. 
For this analysis, the scenario with $\tau \gtrsim 1$ is considered, i.e., it is assumed that all observed cosmic ray sources also emit neutrinos. It is important to mention that in this scenario, the cross section for nuclear disintegration is much larger than the pion production cross section relevant for neutrino production. This means that nuclei are expected to be fully disintegrated before they leave the source, and that ejected cosmic rays are mostly protons.

This paper is organized as follows. The source stacking method is described in Section 2. A discussion about the deflection of UHECRs in magnetic fields is presented in Section 3. The data samples are presented in Section 4 and the background and signal simulations are explained in Section 5. The angular search bin optimization is discussed in Section 6 and the discovery potential in Section 7. The results are given in Section 8 .

\section{SOURCE STACKING METHOD}

Source stacking is a method of noise reduction. The signal adds up linearly with the number of like sources, but the noise goes up roughly with the square root of the sum of squares of background counts around all sources. In other words, the signal-to-noise ratio increases proportionally to the square root of the number of sources. Stacking multiple sources in neutrino astronomy has been an effective way to enhance discovery potential and further constrain astrophysical models (Achterberg et al. 2006; Abbasi et al. 2009a, 2011).

In this analysis, all neutrino candidates observed by the ANTARES telescope in 2007 and 2008, scrambled in right ascension (thus blinding the data set), are considered to be the background. Signal events positioned in the directions of UHECRs observed by the Auger Observatory are added on top of this background in Monte Carlo (MC) simulations. From those simulations, signal counts needed to claim discovery are obtained.

A previously published analysis of the ANTARES telescope observations (Adrián-Martínez et al. 2011, 2012) showed that neither the whole-sky search for hot spots in the neutrino sky nor the search at predefined and astrophysically motivated source positions have led to the discovery of a statistically significant neutrino signal. This, however, does not exclude the possibility that the superposition of weak signals from generically equal sources, each contributing below the individual significance threshold, sums up to a significant signal for that specific source type.

In this analysis, the same data set as in Adrián-Martínez et al. (2011) is used, and the possibility that a class of sources that might emit UHECRs also emit neutrinos is tested. Only the cumulative result for a class of sources is evaluated, not the signal from individual sources.

The ANTARES Collaboration follows a strict blindness policy, i.e., an analysis has to be developed blindly with respect to the data. This prevents statistical fluctuations from affecting the final steps of the analysis and means that no signal is evaluated until the source samples and all analysis parameters are fixed.

\section{MAGNETIC DEFLECTION OF UHECRs}

In this paper, the directions of candidate neutrino events observed by the ANTARES telescope were analyzed for correlations with UHECR events recorded by the Pierre Auger Observatory, using a source stacking method in which the cumulative neutrino signal from all UHECR directions is summed and compared with the expected background. A key parameter for this analysis is the size of the angular search bin around each UHECR direction. The size of this bin is derived from the assumed magnetic deflection of the UHECRs.

The magnitude of magnetic deflection in Galactic and intergalactic magnetic fields is unknown. There are no observational data constraining intercluster magnetic fields, and also, some features of the Galactic magnetic field, such as possible magnetized halo are not yet known. Theoretical predictions of UHECR deflection in both Galactic and intergalactic magnetic fields give various estimates that vary from one to a few tens of degrees, depending on different assumptions.

Protons with the highest energies (above $10^{19} \mathrm{eV}$ ) are expected to be deflected by the Galactic magnetic field up to a few degrees (Stanev 1997; Alvarez-Muñiz et al. 2002; Takami \& Sato 2010). Medina Tanco et al. (1998) calculated that protons with energies of $4 \times 10^{19} \mathrm{eV}$ should be deflected by about $5^{\circ}$. Harari et al. (1999) concluded that $10^{20} \mathrm{eV}$ protons arrive to Earth almost undeflected. Deflection angles of about $3^{\circ}$, for protons of $4 \times 10^{19} \mathrm{eV}$, were estimated by Prouza \& Smida (2003). Since there are no observational data constraining extragalactic magnetic fields, proton deflection in those fields is not known. Simulations by different authors obtained values for an expected proton deflection from a couple to a few tens of degrees (Dolag et al. 2005; Kotera \& Lemoine 2008; Sigl et al. 2004, 2003; Armengaud et al. 2005).

If the composition of UHECRs is mostly heavy, an identification of their sources would likely be impossible, as their expected deflection would then be tens of degrees, and their arrival directions are expected to be isotropically distributed. Medina Tanco et al. (1998) found that Fe nuclei with energies of $2.5 \times 10^{20} \mathrm{eV}$ can be deflected up to $20^{\circ}$ in the Galactic magnetic field. Prouza \& Smida (2003) also calculated deflection angles of a few tens of degrees for heavy UHECRs. This was also confirmed in a recent paper by Takami \& Sato (2010).

However, as it was mentioned earlier, this analysis considers the scenario where most observed UHECRs are protons. The choice of magnetic deflection angle presented in this paper is based on the lower estimates for proton deflection angles, and is further justified by the fact that using larger search bins destroys the benefits of stacking analysis (see Section 6).

\section{NEUTRINO AND UHECR DATA SAMPLES}

The ANTARES neutrino telescope (Ageron et al. 2011) is located in the Mediterranean Sea, $40 \mathrm{~km}$ off the southern coast of France $\left(42^{\circ} 48 \mathrm{~N}, 6^{\circ} 10^{\prime} \mathrm{E}\right)$, at a depth of $2475 \mathrm{~m}$. It was completed in 2008 and its final configuration is a three-dimensional array of photomultipliers in glass spheres (optical modules; Amram et al. 2002), distributed along 12 lines anchored to the sea bottom and kept taut by a buoy at their top. Of these detection lines, 11 contain 25 storeys of triplets of optical modules and one contains 20 triplets. The lines are subject to sea currents and can change shape and orientation. A positioning system based on hydrophones, compasses and tiltmeters is used to monitor the detector geometry with an accuracy of $10 \mathrm{~cm}$. The total instrumented volume of the ANTARES telescope is about $10^{7} \mathrm{~m}^{3}$. The detection principle is based on measuring the Cherenkov light emitted in the detector by high energy muons, that result from neutrino interactions inside or near the instrumented volume of the detector. The large background from downgoing muons produced in cosmic ray air showers is reduced by selecting only upgoing muons as neutrino candidates. 


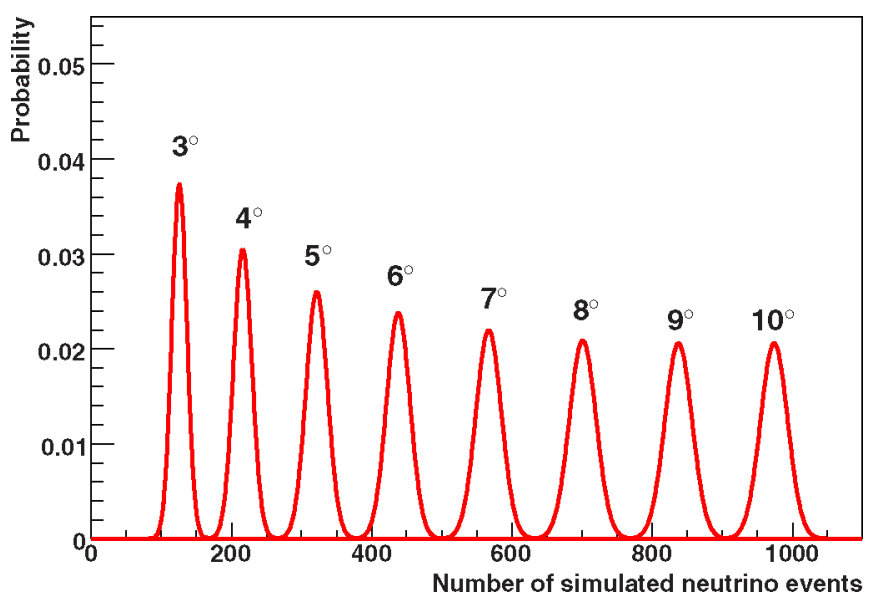

Figure 1. The probability density functions of the number of neutrino events in $3^{\circ}-10^{\circ}$ bins centered on 69 UHECR directions. The corresponding Gaussian mean values (standard deviations) for bins of $3^{\circ}-10^{\circ}$ are $125.8(10.7)$. $216.1(13.3), 321.3(15.6), 437.4(17.1), 566.6(18.0), 700.8(19.1), 838.0(19.1)$ and $974.3(19.0)$, respectively.

(A color version of this figure is available in the online journal.)

The data acquisition system of the detector (Aguilar et al. 2007 ) is based on the "all-data-to-shore" concept, in which signals from the photomultipliers above a given threshold are digitized and sent to shore for processing. The absolute time is provided by GPS and the precise timing resolution for the recorded photo-multiplier tube signals (of the order of $1 \mathrm{~ns}$ ) is required to maintain the angular resolution of the telescope. The arrival times of the hits are calibrated as described in Aguilar et al. (2011a). A L1 hit is defined either as a high-charge hit, or as hits separated by less than 20 ns in optical modules of the same storey. At least five L1 hits are required throughout the detector within a time window of $2.2 \mu \mathrm{s}$, with the relative photon arrival times being compatible with the light coming from a relativistic particle. Independently, events which have L1 hits on two sets of adjacent or next-to-adjacent floors are also selected. The physics events are stored on disk for offline reconstruction.

The data used in this analysis were collected between 2007 January 31, and 2008 December 30. During this time the construction of the detector was still in progress. The detector consisted of 5 lines for most of 2007 and of 9, 10 and 12 lines during 2008. For part of that period, the data acquisition was interrupted for the connection of new lines, and in addition, some periods were excluded due to a high bioluminescenceinduced optical background. The resulting effective live time of the analysis is 304 days.

Triggered events were reconstructed offline to determine the muon trajectory using a multi-stage fitting procedure. The final stage of this procedure consists of a maximum likelihood fit of the measured photon arrival times. A quality parameter, indicated by $\lambda$, is determined based on the final value of the likelihood function. Selection cut on parameter $\lambda>-5.4$ has been optimized in order to obtain the best point source sensitivity (Adrián-Martínez et al. 2011). The estimated angular uncertainty obtained from the muon track fit is required to be smaller than $1^{\circ}$. The final data sample consists of 2190 upgoing neutrino candidate events. For this current analysis, no selection was done based on the energy reconstruction. The angular resolution was estimated to be $0.5 \pm 0.1$. The simulations indicate that the selected sample contains $60 \%$ atmospheric neutrinos, the rest are misreconstructed atmospheric muons.

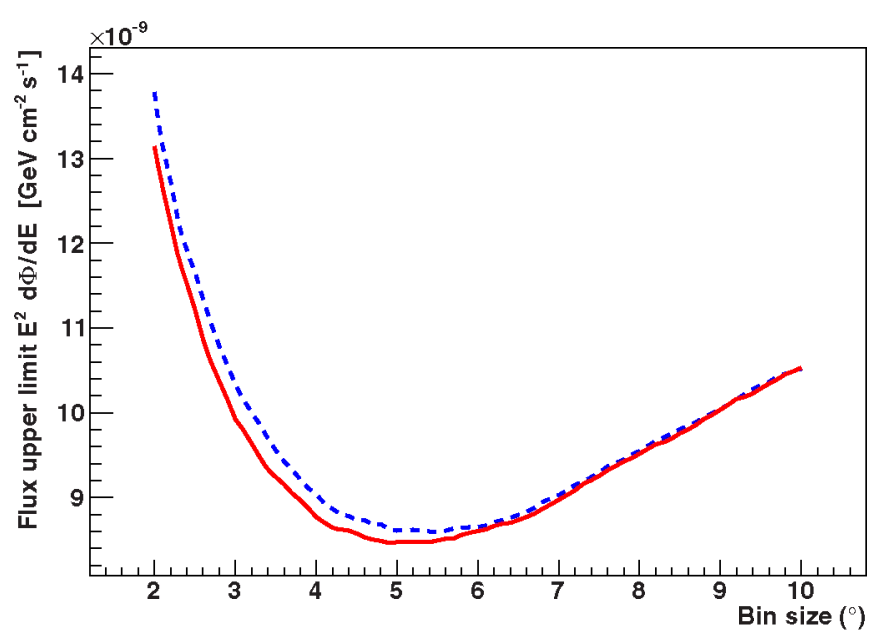

Figure 2. The mean flux upper limit (90\% CL) as a function of the search bin size and for a magnetic deflection of $3^{\circ}$ is presented with the red solid line. The mean upper limit for an angular resolution degraded by a factor two is shown with the blue dashed line.

(A color version of this figure is available in the online journal.)

Previously, the Pierre Auger Observatory reported an anisotropy in the arrival directions of UHECRs (Abraham et al. 2008b) and indicated a correlation with AGNs from the Veron-Cetty \& Veron (VCV) catalog (Véron-Cetty \& Véron 2006). After a scan of the relevant parameters, the prescription was made on a subsample of data and the correlation was found to be the most significant for a sample of 27 events corresponding to cosmic ray energies higher than $57 \mathrm{EeV}$, falling within a bin of size 3.1 around the AGNs from the VCV catalog, located at distances smaller than $75 \mathrm{Mpc}$. However, the HiRes Collaboration reported an absence of a comparable correlation in observations in the Northern hemisphere (Abbasi et al. 2008b). Further, the suggested correlation of the Pierre Auger UHECRs with the nearby AGN sources decreased in a subsequent analysis (Abreu et al. 2010) with 69 events at energies above $55 \mathrm{EeV}$ (10 $\left.0^{19.74} \mathrm{eV}\right)$, observed until 2009 December 31. These 69 UHECR events were used in the correlation analysis presented in this paper. The angular resolution for these events is better than $1^{\circ}$.

\section{BACKGROUND AND SIGNAL SIMULATIONS}

In order to study the statistical significance of any observed correlation between the neutrino and UHECR datasets and determine an optimal angular search bin, an MC set with $10^{6}$ pseudo-experiments is generated, each with 2190 neutrinos and 69 UHECRs. In each of these pseudo-experiments the positions of UHECRs are fixed according to the Pierre Auger Observatory dataset and the neutrino background is randomly generated by scrambling the 2190 neutrinos from the ANTARES telescope dataset in right ascension. The number of neutrinos within an angular bin of chosen size, centered on 69 UHECR events, is counted. The normalized probability density function is calculated and fitted with a Gaussian distribution, to obtain the mean neutrino count and its standard deviation expected from the randomized background samples. This procedure is repeated for a range of different bin sizes.

For illustration, Figure 1 shows an example count of neutrinos for bins of $3^{\circ}-10^{\circ}$ size. The count of events is done by summing neutrinos in all 69 bins for which the minimum angular distance to UHECRs is smaller than the bin size. In this way, when 


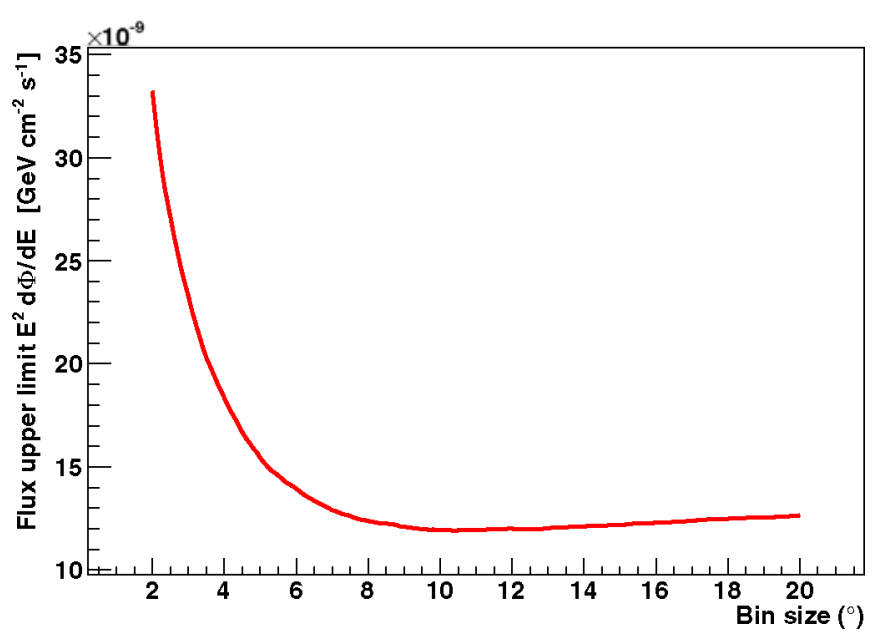

Figure 3. The mean flux upper limit $(90 \% \mathrm{CL})$ as a function of the search bin size and for a magnetic deflection of $6^{\circ}$.

(A color version of this figure is available in the online journal.)

the same neutrino event falls within multiple bins around the UHECRs, a double counting of neutrino events is avoided. After optimizing an angular bin size (as described in Section 6), the significance of the observed number of neutrino events within 69 bins is calculated by comparison with the distribution for the pure background MC sample.

The signal events are simulated assuming a neutrino energy spectrum proportional to $E^{-2}$ and equal flux strength from each of 69 UHECR directions. Flux values from $0.5 \times$ $10^{-8} \mathrm{GeV} \mathrm{cm}^{-2} \mathrm{~s}^{-1}$ to $10^{-7} \mathrm{GeV} \mathrm{cm}^{-2} \mathrm{~s}^{-1}$ are considered. The flux is converted into signal event rate per source using the effective area for 5-12 lines and the corresponding live time. For every source, signal neutrinos are generated according to the Poisson distribution with the event rate per source as mean value. For example, a flux value of $10^{-8} \mathrm{GeV} \mathrm{cm}^{-2} \mathrm{~s}^{-1}$ gives 0.85 signal neutrinos per UHECR source, or about 58 events for all stacked sources. Signal neutrinos are randomly generated according to a Gaussian which is a result of a convolution of the magnetic field deflection window of $3^{\circ}$ and the angular resolution of the ANTARES telescope. The same amount of background neutrinos is removed from a declination band of $10^{\circ}$ centered on each UHECR to ensure that every random sky has 2190 events and to keep the neutrino declination distribution profile close to the observed profile.

\section{ANGULAR SEARCH BIN OPTIMIZATION}

MC predictions are used to obtain an optimal angular search bin size. This is done by the minimization of the mean flux upper limit or so-called Feldman-Cousins sensitivity (Feldman \& Cousins 1998; Hill \& Rawlins 2003) that would be observed over the set of identical experiments with expected background $n_{\mathrm{b}}$ and no true signal.

In such a case, the background $n_{\mathrm{b}}$ fluctuates to different values with different Poisson probabilities, each one associated with an upper limit or the $90 \%$ Feldman-Cousins confidence interval $\mu_{90}$ that is a function of the number of observed events, $n_{\mathrm{obs}}$, and of the expected background $n_{\mathrm{b}}$. Note that this can be done for any level of confidence or any formulation of confidence intervals.

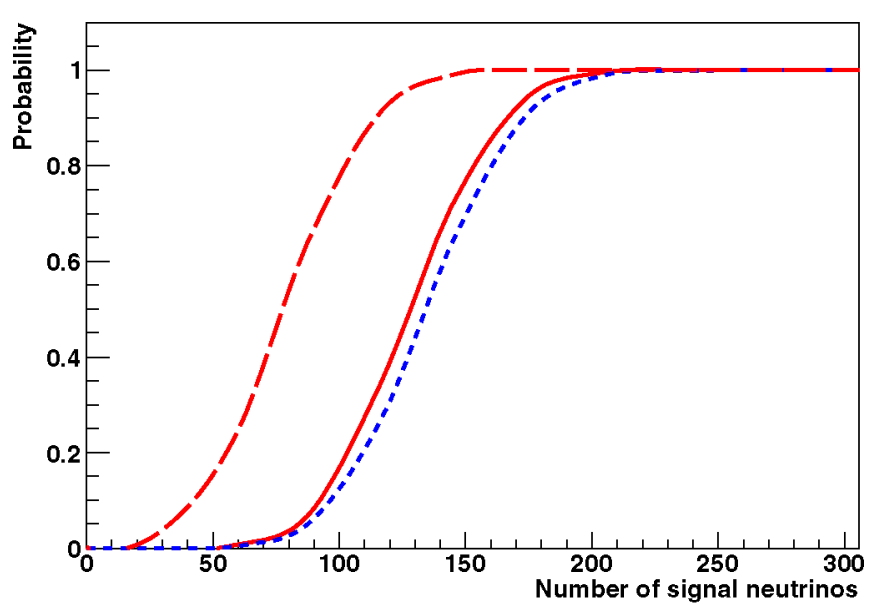

Figure 4. The discovery potential at $3 \sigma$ (red long-dashed line) and $5 \sigma$ (red solid line) $90 \% \mathrm{CL}$ as a function of the number of neutrino signal events from 69 sources on the whole sky. The discovery potential for an angular resolution degraded by a factor two is shown with the blue dashed line.

(A color version of this figure is available in the online journal.)

The mean upper limit is the sum of these expected upper limits, weighted by their Poisson probability of occurrence:

$$
\overline{\mu^{90}}\left(n_{\mathrm{b}}\right)=\sum_{n_{\mathrm{obs}}=0}^{\infty} \mu^{90}\left(n_{\mathrm{obs}}, n_{\mathrm{b}}\right)\left(n_{\mathrm{b}}^{n_{\mathrm{obs}}} /\left(n_{\mathrm{obs}}\right) !\right) e^{-n_{\mathrm{b}}}
$$

Over an ensemble of identical experiments, the strongest constraint on the expected signal flux corresponds to a set of cuts that minimizes the model rejection factor $\mu_{90} / n_{\mathrm{s}}$ and at the same time minimizes the mean flux upper limit that would be obtained over the hypothetical experimental ensemble:

$$
\bar{\Phi}(E, \theta)_{90}=\Phi(E, \theta)\left(\mu_{90} / n_{\mathrm{s}}\right),
$$

where $\Phi(E, \theta)$ is the theoretical flux that is proportional to the number of signal events $n_{\text {sig. }}$. From the equation above, it can be seen that the mean flux upper limit, and as a result also the optimized angular bin size, does not depend on a modeled signal strength.

The described Feldman-Cousin's approach with the Rolke extension (Rolke et al. 2005) was used to calculate the $90 \%$ upper limit on the neutrino flux per source assuming an $E^{-2}$ energy spectrum, as a function of the search bin size.

An $E^{-2}$ energy spectrum was assumed to maintain compatibility with the previously published ANTARES point source search and diffuse limit analysis (Adrián-Martínez et al. 2011, 2012; Aguilar et al. 2011b). This choice was also further discussed and motivated in Aguilar et al. (2011b). The MC simulations showed that $80 \%$ of the signal from neutrino-induced muons, assuming an $E^{-2}$ energy spectrum, will lie in the energy range from 4 to $700 \mathrm{TeV}$. In theoretical predictions, neutrino spectra of $E^{-2}$ or harder are expected (see, e.g., Mannheim 1995; Mannheim et al. 2001; Stecker 2005; Semikoz \& Sigl 2004).

Using $3^{\circ}$ magnetic deflection window, the angular search bin that minimizes the flux upper limit is $4: 9$ (Figure 2). If an assumed magnetic deflection is represented with a Gaussian with a $6^{\circ}$ width, the optimized angular search bin is $10: 4$ (Figure 3 ). Such a large search bin placed around 69 observed UHECRs covers a significant part of the visible sky, destroying the benefit of a stacking analysis. Because of this and in order to avoid the 


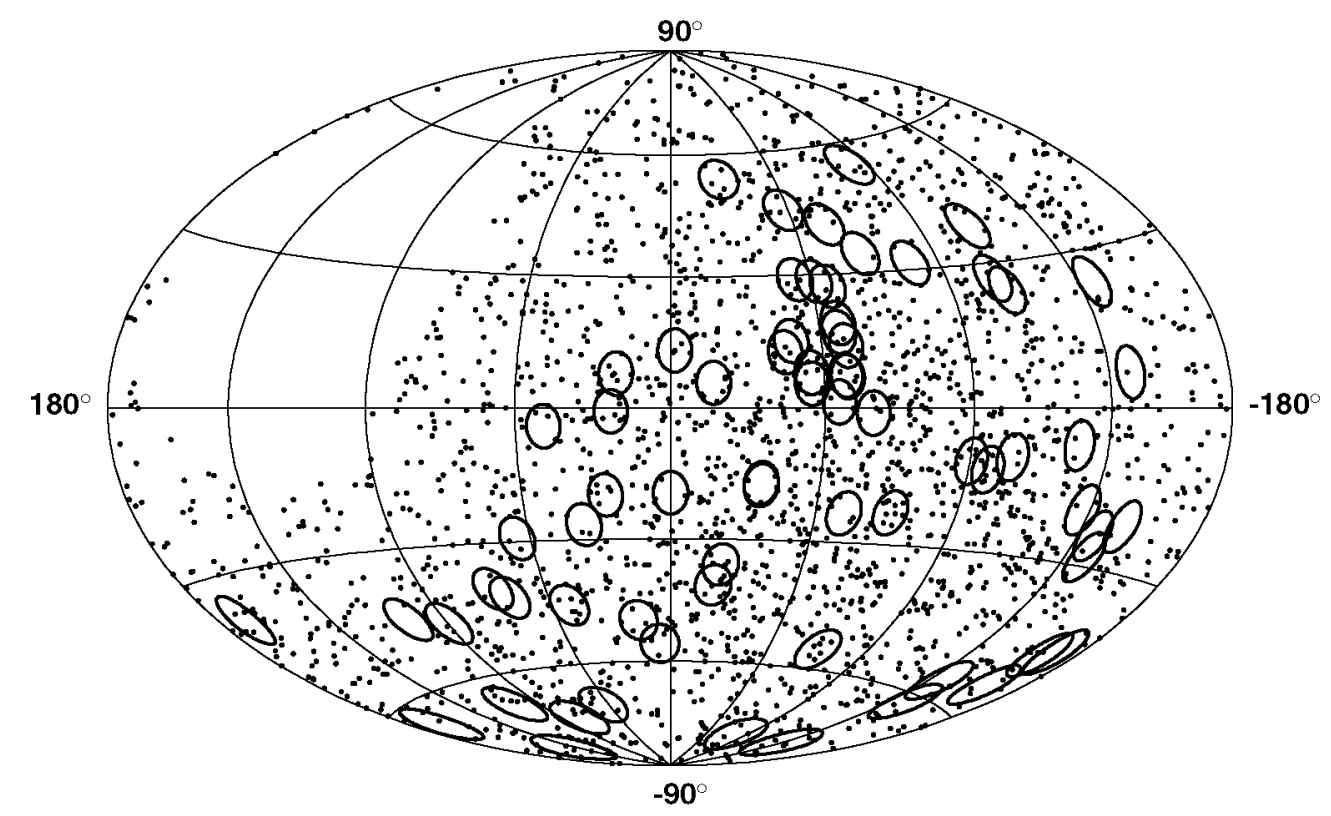

Figure 5. On this sky map in Galactic coordinates, neutrino events are represented with black dots and angular search bins of 4.9 centered on the observed UHECRs with black circles.

trial factor associated with using multiple tolerance windows for the magnetic deflection, a single value of $3^{\circ}$ Gaussian width is adopted for this analysis.

\section{DISCOVERY POTENTIAL}

With the angular search bin size optimized and fixed, it is possible to estimate the probability of making a $3 \sigma$ or a $5 \sigma 90 \% \mathrm{CL}$ discovery given a certain signal flux. First, the neutrino count necessary for a chosen $\sigma$ level is determined from the background MC samples. Then, the number of pseudo-experiments with signal, that have more neutrinos in 69 optimized bins than the chosen $\sigma$ level from background only, is counted and this gives a direct measure of the discovery potential for that particular flux. Figure 4 shows the discovery potential for $5 \sigma$ (red solid line) and $3 \sigma$ (red long-dashed line) discovery, for an optimized bin size of 4.9. Around 125 (75) signal events correlated to the 69 UHECRs directions are needed for a $5 \sigma(3 \sigma)$ discovery in $50 \%$ of trials. This counts correspond to a flux per source of $2.16 \times 10^{-8} \mathrm{GeV} \mathrm{cm}^{-2} \mathrm{~s}^{-1}$ and $1.29 \times 10^{-8} \mathrm{GeV} \mathrm{cm}^{-2} \mathrm{~s}^{-1}$ or the event count per source of 1.8 and 1.1 , respectively.

To quantify the improvement of sensitivity of a source stacking approach, those numbers can be compared to the single source search. To claim a $5 \sigma$ discovery ( $50 \%$ discovery potential), for a localized excess, depending on a declination, 6-10 signal events are needed (Adrián-Martínez et al. 2011, 2012)

To check the effect of the possible angular resolution systematic uncertainty on the sensitivity and discovery potential, MC simulations with an angular resolution degraded by a factor two were performed. The optimized bin value in this case is 5.5 , compared with 4.9 obtained from the observed angular resolution. This degraded angular resolution results in about a $20 \%$ higher neutrino flux upper limit. No significant effect is found on the discovery potential. Figures 2 and 4 show, respectively, optimized bin and discovery potential for observed and two times lower angular resolution. Note that the expected error on the angular resolution, as we already mentioned, is estimated to be much smaller $(0.1)$.

\section{RESULTS}

To analyze the level of correlation between the distribution of 2190 neutrino candidates observed by the ANTARES telescope and $69 \mathrm{UHECRs}$ reported by the Pierre Auger Observatory, the right ascension of the neutrino candidate events was unblinded. The significance of an observed correlation is determined with the help of randomized background samples, using the optimized bin of 4:9. The most probable count for this optimized bin size, or the mean background expectation from the randomized samples, is 310.5 events (in all 69 bins), with a standard deviation of 15.2 events. After unblinding the 2190 ANTARES telescope neutrino candidate events, a count of 290 events within 69 bins is obtained (Figure 5), which is slightly lower than expected. This count is compatible with an underfluctuation of the background, with a significance of $1.4 \sigma$. The corresponding 90\% CL upper limit on the neutrino flux from each observed UHECR direction (assuming an equal flux from all of them and for an $E^{-2}$ energy spectrum) is $5.0 \times 10^{-8} \mathrm{GeV} \mathrm{cm}^{-2} \mathrm{~s}^{-1}$.

None of the 69 angular search bins centered on the observed UHECRs show an individual excess of neutrinos. Individual counts with the largest significances are in the search bins around the observed UHECR with declination of 44.2 and right ascension 224.5 ( 10 events), and with declination of $-50: 6$, and right ascension of $116^{\circ}$ ( 11 events).

The lack of correlation of neutrinos observed by the ANTARES telescope and the UHECRs observed by the Pierre Auger Observatory can be due to various reasons. As already discussed in detail, the magnitude of magnetic deflection that influences the path of UHECRs is not known. Also, UHECRs are only originating from sources within the GZK sphere, while this is not the case with neutrinos. Also, as explained, even if hadronic acceleration is taking place in astrophysical sources, depending on the opacity for protons, they can be emitting both neutrinos and protons, or only neutrinos or protons.

In the future, the increased statistics available from both experiments will allow us to select only the highest energy events thereby reducing the uncertainties related to magnetic deflection. 
The authors acknowledge comments and suggestions of members of the Pierre Auger Collaboration. The authors acknowledge the financial support of the funding agencies: Centre National de la Recherche Scientifique (CNRS), Commissariat á l'énegie atomique et aux énergies alternatives (CEA), Agence National de la Recherche (ANR), Commission Européenne (FEDER fund and Marie Curie Program), Région Alsace (contract CPER), Région Provence-Alpes-Côte d'Azur, Département du Var and Ville de La Seyne-sur-Mer, France; Bundesministerium für Bildung und Forschung (BMBF), Germany; Istituto Nazionale di Fisica Nucleare (INFN), Italy; Stichting voor Fundamenteel Onderzoek der Materie (FOM), Nederlandse organisatie voor Wetenschappelijk Onderzoek (NWO), The Netherlands; Council of the President of the Russian Federation for young scientists and leading scientific schools supporting grants, Russia; National Authority for Scientific Research (ANCS-UEFISCDI), Romania; Ministerio de Ciencia e Innovación (MICINN), Prometeo of Generalitat Valenciana and MultiDark, Spain. We also acknowledge the technical support of Ifremer, AIM and Foselev Marine for the sea operation and the CC-IN2P3 for the computing facilities.

\section{REFERENCES}

Abbasi. R.. Abdou. Y.. Abu-Zayyad. T.. et al. 2011. ApJ. 732. 18 Abbasi, R., Abdou. Y., Ackermann. M., et al. 2009a, ApJL, 701, L47 Abbasi, R. U., Abu-Zayyad, T., Al-Seady, M., et al. 2009b, APh, 32, 53 Abbasi, R. U., Abu-Zayyad, T., Allen, M., et al. 2008a. PhRvL, 100, 101101 Abbasi. R. U.. Abu-Zayyad. T., Allen. M.. et al. 2008b. APh. 30.175 Abraham, J., Abreu, P., Aglietta, M., et al. 2008a. PhRvL, 101, 061101 Abraham, J., Abreu, P., Aglietta, M., et al. 2008b, APh, 29, 188 Abraham. J., Abreu, P., Aglietta, M., et al. 2010, PhLB. 685. 239 Abreu. P.. Aglietta. M.. Ahn. E. J., et al. 2010. APh. 34. 314 Achterberg. A.. Ackermann. M.. Adams. J.. et al. 2006. APh. 26. 282 Adrián-Martínez. S.. Aguilar. J. A.. A1 Samarai. I.. et al. 2011. ApJL. 743. L14 Adrián-Martínez, S., Al Samarai. I., Albert. A.. et al. 2012, ApJ, 760, 53 Ageron, M., Aguilar, J. A., Al Samarai, I., et al. 2011, NIMPA, 656, 11 Aguilar. J. A.. A1 Samarai. I.. Albert. A.. et al. 2011a. APh. 34.539 Aguilar. J. A., A1 Samarai. I., Albert. A., et al. 2011b. PhLB, 696. 16
Aguilar. J. A.. Albert. A.. Ameli. F. et al. 2007. NIMPA. 570. 107 Alvarez-Muñiz. J., Engel. R., \& Stanev, T. 2002, ApJ. 572. 185 Amram, P.. Anghinolfi, M., Anvar, S.. et al. 2002, NIMPA, 484, 369 Armengaud, E., Sigl, G., \& Miniati, F. 2005, PhRvD, 72, 043009 Avrorin, A. V., Aynutdinov, V. M., Balkanov, V. A., et al. 2009. AstL, 35, 651 Bahcall, J., \& Waxman. E. 2001. PhRvD, 64, 023002 Becker, J. K. 2008, PhR, 458, 173

Becker, J. K., \& Biermann. P. L. 2009. APh, 31, 138

Biermann. P. L.. \& Strittmatter, P. A. 1987, ApJ, 322, 643

Dolag, K.. Grasso, D., Springel, V.. \& Tkachev, I. 2005, JCAP. 01, 009

Feldman, G. J.. \& Cousins. R. D. 1998. PhRvD. 57, 3873

Greisen, K. 1966, PhRvL, 16, 748

Harari, D., Mollerach. S., \& Roulet, E. 1999, JHEP, 08, 022

Hill. G. C., \& Rawlins. K. 2003, APh. 19, 393

Kang. H.. \& Jones. T. W. 2005. ApJ. 620. 44

Kang. H., Rachen. J. P., \& Biermann. P. L. 1997, MNRAS. 286. 257

Kang, H., Ryu, D., \& Jones, T. W. 1996, ApJ, 456, 422

Kotera, K., \& Lemoine, M. 2008, PhRvD, 77, 123003

Ma. R.. Ryu. D., \& Kang. H. 2011. JApA. 32. 301

Mannheim. K. 1995. APh, 3. 295

Mannheim, K., Protheroe, R. J., \& Rachen, J. P. 2001, PhRvD, 63, 023003

Medina Tanco, G. A., de Gouveia dal Pino, E. M., \& Horvath, J. E. 1998, ApJ. 492,200

Murase. K., Ioka, K., Nagataki, S., \& Nakamura. T. 2006, ApJL, 651, L5

Nellen. L.. Mannheim. K.. \& Biermann. P. L. 1993, PhRvD. 47. 5270

Prouza, M., \& Smida, R. 2003, A\&A, 410, 1

Rachen, J. P. 2008, Proc. XXth Rencontres de Blois, arXiv:0808.0349

Rachen. J. P., \& Biermann, P. L. 1993, A\&A. 272. 161

Rachen. J. P.. Protheroe, R. J.. \& Mannheim. K. 2000. NuPhS, 80. C812

Rolke. W. A.. López. A. M. \& Conrad. J. 2005. NIMPA. 551. 493

Semikoz, D. V., \& Sigl, G. 2004, JCAP, 04, 003

Sigl, G., Miniati, F., \& Ensslin, T. A. 2003, PhRvD, 68, 043002

Sigl. G.. Miniati. F.. \& Enßlin. T. A. 2004. PhRvD. 70.043007

Stanev, T. 1997. ApJ, 479, 290

Stecker, F. W. 2005, PhRvD, 72, 107301

Stecker, F. W.. Done, C., Salamon, M. H., \& Sommers, P. 1991, PhRvL, 66 2697

Takami. H.. \& Sato. K. 2010. ApJ. 724. 1456

Véron-Cetty. M.-P.. \& Véron. P. 2006, A\&A, 455, 773

Vietri. M. 1995, ApJ, 453, 883

Waxman, E. 1995, PhRvL, 75, 386

Waxman. E., \& Bahcall, J. 1997, PhRvL. 78. 2292

Waxman. E. \& Bahcall. J. 1999. PhRvD. 59. 023002

Zatsepin, Z.. \& Kuzmin, V. 1966, ZhPmR, 4, 144 